\title{
Control Based on Linear Algebra for Trajectory Tracking and Positioning of Second-Order Chained Form System
}

\author{
Leandro Rodriguez, ${ }^{1}$ Emanuel Serrano $\mathbb{i}^{1},{ }^{1}$ Mabel Cristina Sánchez, ${ }^{2}$ \\ and Gustavo Scaglia $\mathbb{D}^{1}$ \\ ${ }^{1}$ National Council of Scientific and Technological Research (CONICET), Instituto de Ingeniería Química, \\ Universidad Nacional de San Juan, Av. Libertador San Martín Oeste, 1109 San Juan, Argentina \\ ${ }^{2}$ Departamento de Ingeniería Química, Universidad Nacional del Sur (UNS), \\ Planta Piloto de Ingeniería Química (PLAPIQUI) (UNS-CONICET), Bahía Blanca 8000, Argentina \\ Correspondence should be addressed to Gustavo Scaglia; gscaglia@unsj.edu.ar
}

Received 22 April 2020; Revised 27 June 2020; Accepted 14 July 2020; Published 1 August 2020

Guest Editor: Yi Qi

Copyright (c) 2020 Leandro Rodriguez et al. This is an open access article distributed under the Creative Commons Attribution License, which permits unrestricted use, distribution, and reproduction in any medium, provided the original work is properly cited.

\begin{abstract}
The development of controllers for underactuated systems with nonholonomic constraints has been a topic of significant interest for many researchers in recent years. These systems are hard to control because their linearization transform them into uncontrollable systems. The proposed approaches involve the use of a permanent excitation in the reference trajectory; coordinate transformation; discontinuities; or complex calculations. This paper proposes the design of the controller of the second-order chained form system for trajectory tracking by using a simpler approach based on linear algebra. Up to the present time, no controllers based on this approach have been designed for that system. The control problem is solved by setting two of the three systems variables as a reference, while the remaining variable is calculated imposing the condition that the equations system has an exact solution to ensure that tracking errors go to zero. The stability of the proposed controller is theoretically demonstrated, and simulations results show a suitable control system performance. Also, no coordinate transformation is necessary.
\end{abstract}

\section{Introduction}

In recent years, the interest in the control of underactuated systems with nonholonomic constraints has increased [1]. Underactuated systems are mechanical systems with fewer control inputs than degrees of freedom and nonintegrable acceleration constraints [2]. This paper considers the control for trajectory tracking and positioning of second-order chained form systems. These are hard to control because their linearization transform them into uncontrollable systems. The well-known paper of Brockett highlights the difficulty of solving these problems [3].

Several articles addressed the control of underactuated systems with nonholonomic constraints. Aneke et al. [4, 5] resolved the problem of trajectory tracking by using a threestage method. In the first step, they transformed the original system into an extended chained form system with a cascade arrangement. They applied a linear feedback controller to the first subsystem. After that, the second subsystem is exponentially stabilized by employing a backstepping procedure. In the third step, they showed the closed-loop tracking dynamics stability of the system. More recently, other authors used the backstepping procedure to develop control methodologies for trajectory tracking $[6,7]$. A permanent excitation in the reference trajectory is also necessary for system stability. This implies that the signals must not converge to zero, limiting their applications.

Also, in [8], smooth time-varying controllers are proposed to address the exponential tracking of second-order nonholonomic systems. These techniques use coordinate transformation based on time-varying dilation with a cascade-design strategy. In contrast to the previous approach, it does not require the excitation condition in the reference trajectory. This kind of control laws frequently involves 
complex calculations; their design is not intuitive and only applies for special nonholonomic systems. Another method used a discontinuous coordinate transformation to map the initial system into the space of discontinuous nonholonomic systems. In this new space, the control problem can be solved $[9,10]$.

An adaptive dynamic sliding mode controller with integrator was also found in the literature to control nonholonomic systems [11]. This scheme uses a modified backstepping kinematic controller for generating the kinematic trajectories and the velocity errors to drive the sliding surface. The normalized least-square mean was used to control the switching of a sliding mode controller in the presence of uncertainties and parameter variations. Also, other authors developed sliding mode control methodologies for trajectory tracking and positioning of nonholonomic second-order chained form systems [12-14]. Sarfraz and ur Rehman [15] addressed the positioning problem of a particular nonholonomic planar underactuated mechanical system using adaptive sliding mode control. The control law assures the derivative of a suitable Lyapunov function becomes strictly negative.

The aforementioned controllers use a permanent excitation in the reference trajectory $[4,5]$; coordinate transformation [8]; discontinuities [9, 10]; and high computational load calculations [11-15] to solve the trajectory tracking problem. To overcome these drawbacks, this paper aims to solve the problem of trajectory tracking of a second-order chained form system with nonholonomic constraints by using a novel control law inspired by the design method Linear Algebra-Based Controller (LABC) [16]. This technique computes the control actions by solving a system of linear equations and has been applied to different nonlinear systems [17-23].

Until now, LABC has not been applied to second-order chained form systems with nonholonomic constraints in the space of the original variables. For this reason, the major contribution of this work is to extend the LABC technique to solve the tracking and positioning problem for these systems. The system should follow the reference coordinates of two of three variables while the other one is unknown. The key point of this strategy is to find the conditions for which the system of linear equations has an exact solution, ensuring the calculus of the remaining variable, named sacrificed variable. In this way, the reference trajectories of the selected variables and the sacrificed one form an admissible trajectory of the system and the tracking error goes to zero [16].

This paper is organized as follows. The Materials and Methods section describes the second-order chained form system with nonholonomic constraints and solves the problem of trajectory tracking and positioning using a controller based on linear algebra. The stability of the closedloop system is demonstrated by applying the Khalil lemma. In the Results and Discussion section, simulation results show the effectiveness of the proposed controllers. Conclusions are given at the end of this manuscript.

\section{Materials and Methods}

2.1. Problem Formulation. This section presents the special canonical form of an underactuated system with nonholonomic constraints. The control of this system remains a problem under study [1]. One of the most important issues is the transformation to a chained form.

Consider the second-order chained form system, $S_{\xi}$, given by following set of equations:

$$
\begin{aligned}
& \ddot{\xi}_{1}=u_{1}, \\
& \ddot{\xi}_{2}=u_{2}, \\
& \ddot{\xi}_{3}=\xi_{2} u_{1},
\end{aligned}
$$

where $\xi=\left[\xi_{1} ; \xi_{2} ; \xi_{3}\right]^{T} \in R^{3}$ are the generalized coordinates of the second-order chained form system and $\mathbf{u}=\left[u_{1} u_{2}\right]^{T}$ represents the inputs such that $u_{1} \in R$ and $u_{2} \in R$. Also, the reference trajectory is $\Phi_{\xi}=\left[\xi_{1, \mathrm{ref}} ; \xi_{2, \mathrm{ref}} ; \xi_{3, \mathrm{ref}}\right]$.

The tracking errors $\mathbf{e}=\left[e_{1} ; e_{2} ; e_{3}\right]^{T} \in R^{3}$ are the differences between the reference paths and the variables:

$$
\begin{aligned}
& e_{1}=\xi_{1, \mathrm{ref}}-\xi_{1}, \\
& e_{2}=\xi_{2, \mathrm{ref}}-\xi_{2}, \\
& e_{3}=\xi_{3, \mathrm{ref}}-\xi_{3} .
\end{aligned}
$$

This work proposes the design of a controller for trajectory tracking and positioning of a second-order nonholonomic system represented by (1)-(3).

2.2. Problem 1: Trajectory Tracking. Given the system $S_{\xi}$, the trajectory tracking control problem is solvable if it is possible to design an appropriate controller of the form

$$
\begin{aligned}
& u_{1}=u_{1}\left(t, \xi, \Phi_{\xi}\right), \\
& u_{2}=u_{2}\left(t, \xi, \Phi_{\xi}\right),
\end{aligned}
$$

such that the closed-loop system constituted by (1)-(3) and the control actions (5) and (6) is globally uniformly asymptotically stable.

2.3. Problem 2: Positioning. Given the system $S_{\xi}$, the positioning control problem is solvable if it is possible to design an appropriate controller of the form

$$
\begin{aligned}
& u_{1}=u_{1}\left(t, \xi, \Phi_{\xi}\right), \\
& u_{2}=u_{2}\left(t, \xi, \Phi_{\xi}\right),
\end{aligned}
$$

such that the closed-loop system constituted by (1)-(3) and the control actions (7) and (8) is globally uniformly and asymptotically stable. Here, the reference trajectory $\Phi_{\xi}$ is zero.

To the best of our knowledge, LABC technique has not been applied directly to systems of second-order equations without considering a model in state variables; therefore, we extend its use to these systems. The control method is based on determining the conditions for which the system has an exact solution. Knowing the system model, it is only necessary to fix the desired trajectory of $\xi_{1}\left(\xi_{1, \text { ref }}\right)$ and $\xi_{3}\left(\xi_{3, \text { ref }}\right)$ to find the solution [16]. 
2.4. Controller Design for Trajectory Tracking. Given an initial state $\xi(0)$, the system represented by (1)-(3), $S_{\xi}$, should follow the desired trajectory $\Phi_{\xi}$ with a low tracking error. Therefore, it is necessary to find a control law capable of generating a signal $\mathbf{u}=\left[u_{1} u_{2}\right]^{T}$ such that it satisfies this objective.

The system $S_{\xi}$ is expressed in matrix form as follows:

$$
\left[\begin{array}{ll}
1 & 0 \\
0 & 1 \\
\xi_{2} & 0
\end{array}\right]\left[\begin{array}{l}
u_{1} \\
u_{2}
\end{array}\right]=\left[\begin{array}{l}
\ddot{\xi}_{1} \\
\ddot{\xi}_{2} \\
\ddot{\xi}_{3}
\end{array}\right] .
$$

To calculate the control actions $u_{1}$ and $u_{2}$, (9) should have an exact solution, that is,

$$
\begin{gathered}
u_{2}=\ddot{\xi}_{2}, \\
{\left[\begin{array}{l}
1 \\
\xi_{2}
\end{array}\right] u_{1}=\left[\begin{array}{l}
\ddot{\xi}_{1} \\
\ddot{\xi}_{3}
\end{array}\right] .}
\end{gathered}
$$

If a proportional control approach is assumed, the tracking errors are the differences between the reference and real trajectories. Thus, the second-order derivatives of the variables $\xi_{1}$ and $\xi_{3}$, i.e., $\ddot{\xi}_{1}$ and $\ddot{\xi}_{3}$, are $\ddot{\xi}_{1}=\ddot{\xi}_{1, \text { ref }}+k_{11}\left(\dot{\xi}_{1 \text {,ref }}-\right.$ $\left.\dot{\xi}_{1}\right)+k_{10}\left(\xi_{1, \text { ref }}-\xi_{1}\right)$ and $\dot{\xi}_{3}=\ddot{\xi}_{3, \text { ref }}+k_{31}\left(\dot{\xi}_{3, \text { ref }}-\xi_{3}\right)+$ $k_{30}\left(\xi_{3, \text { ref }}-\xi_{3}\right)$, respectively [16].

If the internal variable $\xi_{2 \text {,ez }}$ is set to the value of $\xi_{2}$ for which the above system has an exact solution, then (11) is rewritten as

$$
\left[\begin{array}{c}
1 \\
\xi_{2, \mathrm{ez}}
\end{array}\right] u_{1}=\left[\begin{array}{l}
\ddot{\xi}_{1, \mathrm{ref}}+k_{11}\left(\dot{\xi}_{1, \mathrm{ref}}-\dot{\xi}_{1}\right)+k_{10}\left(\xi_{1, \mathrm{ref}}-\xi_{1}\right) \\
\ddot{\xi}_{3, \mathrm{ref}}+k_{31}\left(\dot{\xi}_{3, \mathrm{ref}}-\dot{\xi}_{3}\right)+k_{30}\left(\xi_{3, \mathrm{ref}}-\xi_{3}\right)
\end{array}\right],
$$

and the tracking errors in variables 1 and 3 tend to zero.

Variable $\xi_{2, \text { ez }}$ is computed according to

$$
\xi_{2, \mathrm{ez}}=\frac{\ddot{\xi}_{3, \text { ref }}+k_{31}\left(\dot{\xi}_{3, \mathrm{ref}}-\dot{\xi}_{3}\right)+k_{30}\left(\xi_{3, \mathrm{ref}}-\xi_{3}\right)}{\ddot{\xi}_{1, \mathrm{ref}}+k_{11}\left(\dot{\xi}_{1, \mathrm{ref}}-\dot{\xi}_{1}\right)+k_{10}\left(\xi_{1, \mathrm{ref}}-\xi_{1}\right)} \text {. }
$$

Remark 1. Variable $\xi_{2}$ follows $\xi_{2, \text { ez }}$ and this one tracks $\xi_{2, \text { ref }}$. The first-order derivatives of the tracking errors are $\dot{e}_{1}=$ $\dot{\xi}_{1, \text { ref }}-\dot{\xi}_{1}$ and $\dot{e}_{3}=\dot{\xi}_{3 \text {,ref }}-\dot{\xi}_{3}$, and the control action $u_{1}$ is obtained using the linear least-squares approximation method to (12) is

$$
u_{1}=\frac{\ddot{\xi}_{1, \mathrm{ref}}+k_{11} \dot{e}_{1}+k_{10} e_{1}+\xi_{2, \mathrm{ez}}\left(\ddot{\xi}_{3, \mathrm{ref}}+k_{31} \dot{e}_{3}+k_{30} e_{3}\right)}{1+\xi_{2, \mathrm{ez}}^{2}} .
$$

We can write the second-order derivative of the variable $\xi_{2}\left(\ddot{\xi}_{2}\right)$ as a function of the sacrificed variable $\xi_{2, \text { ez }}$ :

$$
\ddot{\xi}_{2}=\ddot{\xi}_{2, \mathrm{ez}}+k_{21}\left(\dot{\xi}_{2, \mathrm{ez}}-\dot{\xi}_{2}\right)+k_{20}\left(\xi_{2, \mathrm{ez}}-\xi_{2}\right) .
$$

Furthermore, the first-order derivative of the tracking error of variable 2 is $\dot{e}_{2}=\dot{\xi}_{2, \text { ez }}-\dot{\xi}_{2}$, and the control action $u_{2}$ is

$$
u_{2}=\ddot{\xi}_{2, \mathrm{ez}}+k_{21} \dot{e}_{2}+k_{20} e_{2}
$$

Remark 2. If the numerator and denominator of (13) tend to zero simultaneously, the division is undetermined and $\xi_{2, \text { ez }}$ cannot be calculated. To avoid this problem, it is necessary to choose adequately the coefficients $k_{11}, k_{10}, k_{31}$, and $k_{30}$ forcing the errors $e_{3}$ and $\dot{e}_{3}$ to go to zero more quickly than $e_{1}$ and $\dot{e}_{1}$. This avoids that $\xi_{2, \text { ez }}$ tends to infinity following trajectories whose second derivatives are zero (linear and constant reference trajectories).

2.5. Stability of the Tracking Error Dynamics: Trajectory Tracking Case. In this section, the stability of the closed-loop system $[(1)-(3),(14)-(16)]$ is demonstrated.

Theorem 1. Given the second-order chained form system $S_{\xi}$, expressed in (1)-(3), and the control actions $u_{1}$ and $u_{2}$ formulated by (14)-(16), $\sqrt{e_{1}^{2}+e_{2}^{2}+e_{3}^{2}} \longrightarrow 0$ when $t \longrightarrow \infty$.

Proof. The following steps are proposed to prove Theorem 1 .

\section{Step 1}

Variable $\xi_{2, \text { ez }}$ is substituted in (14), and after performing algebraic manipulations, $u_{1}$ is obtained:

$$
\begin{gathered}
u_{1}=\frac{\left(\left(\ddot{\xi}_{1, \mathrm{ref}}+k_{11} \dot{e}_{1}+k_{10} e_{1}\right)^{2}+\left(\ddot{\xi}_{3, \mathrm{ref}}+k_{31} \dot{e}_{3}+k_{30} e_{3}\right)^{2}\right) /\left(\ddot{\xi}_{1, \mathrm{ref}}+k_{11} \dot{e}_{1}+k_{10} e_{1}\right)}{\left(\left(\ddot{\xi}_{1, \mathrm{ref}}+k_{11} \dot{e}_{1}+k_{10} e_{1}\right)^{2}+\left(\ddot{\xi}_{3, \mathrm{ref}}+k_{31} \dot{e}_{3}+k_{30} e_{3}\right)^{2}\right) /\left(\ddot{\xi}_{1, \mathrm{ref}}+k_{11} \dot{e}_{1}+k_{10} e_{1}\right)^{2}}, \\
u_{1}=\ddot{\xi}_{1, \mathrm{ref}}+k_{11} \dot{e}_{1}+k_{10} e_{1} .
\end{gathered}
$$

From (1), $u_{1}=\ddot{\xi}_{1}$, also we define the second-order derivative of the tracking error of variable 1 as $\ddot{e}_{1}=\left(\ddot{\xi}_{1, \text { ref }}-\ddot{\xi}_{1}\right)$; in consequence, (18) is rewritten as follows: 


$$
0=\ddot{e}_{1}+k_{11} \dot{e}_{1}+k_{10} e_{1} \text {. }
$$

Step 2

Replacing (16) with (10),

$$
0=\ddot{\xi}_{2, \mathrm{ez}}-\ddot{\xi}_{2}+k_{21} \dot{e}_{2}+k_{20} e_{2},
$$

and defining the second-order derivative of the tracking error of variable 2 as $\ddot{e}_{2}=\left(\ddot{\xi}_{2, \mathrm{ez}}-\ddot{\xi}_{2}\right)$, the following equation arises:

$$
0=\ddot{e}_{2}+k_{21} \dot{e}_{2}+k_{20} e_{2} \text {. }
$$

Step 3

Given that $\xi_{2}=\left(\xi_{2, \mathrm{ez}}-e_{2}\right)$, the algebraic manipulation of (3) gives

$$
\ddot{\xi}_{3}=\left(\xi_{2, \mathrm{ez}}-e_{2}\right) u_{1} .
$$

Furthermore, from (13) and (14), the following expression is obtained: $\quad \xi_{2, \text { ez }} u_{1}=\ddot{\xi}_{3, \text { ref }}+k_{31}\left(\dot{\xi}_{3, \text { ref }}-\dot{\xi}_{3}\right)+$ $k_{30}\left(\xi_{3 \text {,ref }}-\xi_{3}\right)$. We define the second-order derivative of the tracking error of variable 3 as $\ddot{e}_{3}=\ddot{\xi}_{3}-\ddot{\xi}_{3 \text {,ref }}$ and rewrite (22) as

$$
0=\ddot{e}_{3}+k_{31}\left(\dot{\xi}_{3, \text { ref }}-\dot{\xi}_{3}\right)+k_{30}\left(\xi_{3, \text { ref }}-\xi_{3}\right)-e_{2} u_{1} .
$$

By replacing the control action $u_{1}=\ddot{\xi}_{1 \text {,ref }}+k_{11} \dot{e}_{1}+k_{10} e_{1}$ in the above equation, the following second-order equation arises:

$$
\begin{aligned}
0= & \ddot{e}_{3}+k_{31}\left(\dot{\xi}_{3, \mathrm{ref}}-\dot{\xi}_{3}\right)+k_{30}\left(\xi_{3, \mathrm{ref}}-\xi_{3}\right) \\
& -e_{2}\left(\ddot{\xi}_{1, \mathrm{ref}}+k_{11} \dot{e}_{1}+k_{10} e_{1}\right) .
\end{aligned}
$$

Then, (19), (21), and (24) are rewritten according to (5.32) and (5.33) on page 221 of [24], where $\dot{x}_{1}=f_{1}\left(t, x_{1}, x_{2}\right)$ and $\dot{x}_{2}=f_{1}\left(t, x_{2}\right)$, being $\mathbf{x}_{1}=\left\{\dot{\xi}_{1} ; \xi_{1} ; \dot{\xi}_{3}\right.$; $\left.\xi_{3}\right\}$ and $\mathbf{x}_{2}=\left\{\dot{\xi}_{2} ; \xi_{2}\right\}$. By defining $x_{11}=e_{1} ; x_{12}=\dot{e}_{1} ; x_{21}=e_{2}$; $x_{22}=\dot{e}_{2} ; x_{13}=e_{3} ; x_{14}=\dot{e}_{3}$, the following system of equations is obtained:

$$
\begin{aligned}
& {\left[\begin{array}{l}
\dot{x}_{11} \\
\dot{x}_{12} \\
\dot{x}_{13} \\
\dot{x}_{14}
\end{array}\right]=\left[\begin{array}{cccc}
0 & 1 & 0 & 0 \\
-k_{10} & -k_{11} & 0 & 0 \\
0 & 0 & 0 & 1 \\
0 & 0 & -k_{30} & -k_{31}
\end{array}\right]\left[\begin{array}{l}
x_{11} \\
x_{12} \\
x_{13} \\
x_{14}
\end{array}\right]} \\
& -x_{21}\left[\begin{array}{c}
0 \\
0 \\
0 \\
\ddot{\xi}_{1, \mathrm{ref}}+k_{11} \dot{e}_{1}+k_{10} e_{1}
\end{array}\right] \text {, } \\
& {\left[\begin{array}{l}
\dot{x}_{21} \\
\dot{x}_{22}
\end{array}\right]=\left[\begin{array}{cc}
0 & 1 \\
-k_{20} & -k_{21}
\end{array}\right]\left[\begin{array}{l}
x_{21} \\
x_{22}
\end{array}\right] \text {. }}
\end{aligned}
$$

Finally, by applying Lemma 5.6 of Khalil, the system is globally uniformly asymptotically stable and satisfies the condition $\sqrt{e_{1}^{2}+e_{2}^{2}+e_{3}^{2}} \longrightarrow 0$ when $\longrightarrow \infty$.

Remark 3. If the controller parameters are adjusted such that the roots of the polynomial (19), $\ddot{e}_{1}+k_{11} \dot{e}_{1}+k_{10} e_{1}=0$, have a negative real part closer to the imaginary axis than the roots of the polynomial (24), $\ddot{e}_{3}+k_{31} \dot{e}_{3}+k_{30} e_{3}=0$, then $e_{3}$ will go to zero faster than $e_{1}$ and $\xi_{2, \text { ez }}$ does not go to infinity.

2.6. Controller Design for Positioning. The positioning problem for the system, $S_{\xi}$, consists in computing the control inputs, $u_{1}=u_{1}\left(t, \xi, \Phi_{\xi}\right)$ and $u_{2}=u_{2}\left(t, \xi, \Phi_{\xi}\right)$, such that the origin of the system is stable for any initial state $\xi(0)$. Positioning is a particular case of trajectory tracking, where $\dot{\xi}_{1, \text { ref }}, \dot{\xi}_{3, \text { ref }}, \ddot{\xi}_{1, \text { ref }}$, and $\ddot{\xi}_{3, \text { ref }}$ are zero, and $\xi_{2, \text { ez }}$ is the sacrificed variable.

The system $S_{\xi}$ is expressed in matrix form as follows:

$$
\left[\begin{array}{ll}
1 & 0 \\
0 & 1 \\
\xi_{2} & 0
\end{array}\right]\left[\begin{array}{l}
u_{1} \\
u_{2}
\end{array}\right]=\left[\begin{array}{l}
\ddot{\xi}_{1} \\
\ddot{\xi}_{2} \\
\ddot{\xi}_{3}
\end{array}\right] .
$$

To calculate the control actions $u_{1}$ and $u_{2}$, (26) should have an exact solution; that is,

$$
\begin{aligned}
& u_{2}=\ddot{\xi}_{2}, \\
& {\left[\begin{array}{l}
1 \\
\xi_{2}
\end{array}\right] u_{1}=\left[\begin{array}{l}
\ddot{\xi}_{1} \\
\ddot{\xi}_{3}
\end{array}\right] . }
\end{aligned}
$$

If a proportional control approach is assumed, the tracking errors are the differences between the reference (zero) and real trajectories. Thus, the variables $\ddot{\xi}_{1}$ and $\ddot{\xi}_{3}$ are $\ddot{\xi}_{1}=-k_{11} \dot{\xi}_{1}+k_{10} e_{1}$ and $\ddot{\xi}_{3}=-k_{31} \dot{\xi}_{3}+k_{30} e_{3}$.

If $\xi_{2, \text { ez }}$ is the value of $\xi_{2}$ for which the above system has an exact solution, then

$$
\left[\begin{array}{c}
1 \\
\xi_{2, \mathrm{ez}}
\end{array}\right] u_{1}=\left[\begin{array}{c}
-k_{11} \dot{\xi}_{1}+k_{10} e_{1} \\
-k_{31} \dot{\xi}_{3}+k_{30} e_{3}
\end{array}\right],
$$

and the tracking errors in variables 1 and 3 tend to zero.

Variable $\xi_{2, \text { ez }}$ is computed according to

$$
\xi_{2, \mathrm{ez}}=\frac{-k_{31} \dot{\xi}_{3}+k_{30} e_{3}}{-k_{11} \dot{\xi}_{1}+k_{10} e_{1}}
$$

Remark 4. Variable $\xi_{2}$ follows $\xi_{2 \text {,ez }}$ and this one tracks $\xi_{2 \text {,ref }}$.

The control action $u_{1}$ obtained using linear least-squares approximation method to (28) is

$$
u_{1}=\frac{-k_{11} \dot{\xi}_{1}+k_{10} e_{1}+\xi_{2, \mathrm{ez}}\left(-k_{31} \dot{\xi}_{3}+k_{30} e_{3}\right)}{1+\xi_{2, \mathrm{ez}}^{2}} .
$$

Furthermore, the control action $u_{2}$ is a function of variables $\ddot{\xi}_{2, \mathrm{ez}}, \dot{e}_{2}$, and $e_{2}$ :

$$
u_{2}=\ddot{\xi}_{2, \mathrm{ez}}+k_{21} \dot{e}_{2}+k_{20} e_{2} .
$$


Remark 5. Equations (30) and (31) for positioning are a particular case of (13) and (14) for trajectory tracking of the system, $S_{\xi}$.

2.7. Stability of the Tracking Error Dynamics: Positioning Case. In this section, the stability of the closed-loop system $[(1)-(3)$ and (29)-(31)] is demonstrated.

Theorem 2. Given the second-order chained form system (1)-(3), and the control actions $u_{1}$ and $u_{2}$ formulated in (29)-(31), the variables $\xi_{1}, \xi_{2}, \xi_{3} \longrightarrow 0$ and $\xi_{2, e z}, \dot{\xi}_{2, e z}$, $\dot{\xi}_{2, e z} \longrightarrow 0$ when $t \longrightarrow \infty$.

Proof. The following steps are proposed to prove Theorem 2:

Step 1

Variable $\xi_{2, \text { ez }}$ is substituted in (30), and after doing algebraic manipulations, $u_{1}$ is obtained:

$$
u_{1}=-k_{11} \dot{\xi}_{1}+k_{10} e_{1}
$$

From (1), $u_{1}=\ddot{\xi}_{1}$. Then, (32) is rewritten as follows:

$$
0=-\ddot{\xi}_{1}-k_{11} \dot{\xi}_{1}+k_{10} e_{1} \text {. }
$$

Step 2

Substituting... (31) with (10), and given that $\ddot{e}_{2}=\left(\ddot{\xi}_{2, \text { ez }}-\ddot{\xi}_{2}\right)$, the following equation arises:

$$
0=\ddot{e}_{2}+k_{21} \dot{e}_{2}+k_{20} e_{2} .
$$

Equation (34) for positioning is coincident with (21) for trajectory tracking.

Step 3

Given that $\xi_{2}=\left(\xi_{2, \text { ez }}-e_{2}\right)$, the algebraic manipulation of (3) gives

$$
\ddot{\xi}_{3}=\left(\xi_{2, \text { ez }}-e_{2}\right) u_{1} .
$$

Furthermore, from (29) and (30), the following expression is obtained: $\xi_{2, e z} u_{1}=-k_{31} \dot{\xi}_{3}-k_{30} \xi_{3}$. We rewrite (35) as

$$
0=\ddot{\xi}_{3}-k_{31} \dot{\xi}_{3}-k_{30} \xi_{3}-e_{2}\left(-k_{11} \dot{\xi}_{1}+k_{10} e_{1}\right) .
$$

Then, (33), (34), and (36) for positioning are rewritten according ..to (5.32) and (5.33) of [24]. where $\dot{\xi}_{1, \text { ref }}=0$; $\dot{\xi}_{3, \text { ref }}=0 ; \ddot{\xi}_{1, \text { ref.. }}=0$; and $\ddot{\xi}_{3, \text { ref. }}=0 ; \dot{e}_{1}=-\dot{\xi}_{1} ; \dot{e}_{3}=-\dot{\xi}_{3} ; \ddot{e}_{1}=$ $-\xi_{1}$ and $\ddot{e}_{3}=-\ddot{\xi}_{3}$, being $\mathbf{x}_{1}=\left\{\dot{\xi}_{1} ; \xi_{1} ; \dot{\xi}_{3} ; \xi_{3}\right\}$ and $\mathbf{x}_{2}=\left\{\xi_{2} ; \xi_{2}\right\}$. By defining $x_{11}=e_{1} ; x_{12}=\dot{e}_{1} ; x_{21}=e_{2} ; x_{22}=\dot{e}_{2} ; x_{13}=e_{3}$; and $x_{14}=\dot{e}_{3}$, the following equations arise:

$$
\begin{aligned}
& {\left[\begin{array}{l}
\dot{x}_{11} \\
\dot{x}_{12} \\
\dot{x}_{13} \\
\dot{x}_{14}
\end{array}\right]=\left[\begin{array}{cccc}
0 & 1 & 0 & 0 \\
-k_{10} & -k_{11} & 0 & 0 \\
0 & 0 & 0 & 1 \\
0 & 0 & -k_{30} & -k_{31}
\end{array}\right]\left[\begin{array}{l}
x_{11} \\
x_{12} \\
x_{13} \\
x_{14}
\end{array}\right]} \\
& -x_{21}\left[\begin{array}{c}
0 \\
0 \\
0 \\
k_{11} \dot{e}_{1}+k_{10} e_{1}
\end{array}\right] \text {, } \\
& {\left[\begin{array}{l}
\dot{x}_{21} \\
\dot{x}_{22}
\end{array}\right]=\left[\begin{array}{cc}
0 & 1 \\
-k_{20} & -k_{21}
\end{array}\right]\left[\begin{array}{l}
x_{21} \\
x_{22}
\end{array}\right] \text {. }}
\end{aligned}
$$

Finally, according to the stability lemma of Khalil [24], the system is globally uniformly asymptotically stable and verifies the conditions $\left(\xi_{1}, \xi_{2}, \xi_{3} \longrightarrow 0\right)$ and $\left(\xi_{2, \text { ez }}, \dot{\xi}_{2, \text { ez }}\right.$, $\ddot{\xi}_{2, \mathrm{ez}} \longrightarrow 0$ ) when $t \longrightarrow \infty$.

Remark 6. By adjusting the controller parameters in such a way the roots of the polynomial (33) have a negative real part closer to the imaginary axis than the roots of the polynomial (36), the tracking error $e_{3}$ will go to zero faster than $e_{1}$ and $\xi_{2, \text { ez }}$ will not tend to infinity.

\section{Results and Discussion}

The closed-loop system (1)-(3) and (13), (14), (16) for trajectory tracking is simulated and analyzed in the first test. In the same way, the closed-loop system (1)-(3) and (29)-(31) for positioning is simulated, and the results are discussed in the second test.

3.1. Trajectory Tracking Test. The aim of this section is to show that given a reference trajectory, the proposed controller can calculate a control law that allows following effectively. The reference trajectories of $\xi_{1} ; \xi_{2}$; and $\xi_{3}$ are simulated considering $\xi_{1}(0)=40$ and $\xi_{2}(0)=\xi_{3}(0)=0$ as the initial state of the system, and $\xi_{1 \text {,ref }}$ and $\xi_{3 \text {,ref follow a }}$ parabolic path, while $\xi_{2 \text {,ref }}$ follows an ascending sine wave. The controller parameters, set by trial and error, are $k_{10}=0.72 ; k_{11}=0.72 ; k_{20}=0.55 ; k_{21}=0.55 ; k_{30}=0.90 ;$ and $k_{31}=0.90$. With these values, $\xi_{1} ; \xi_{2}$; and $\xi_{3}$ converge satisfactorily to the reference trajectory. Figure 1 shows the trajectory of variable 1 . Variable $\xi_{1}$ (blue line) initially fluctuates around its reference $\xi_{1, \text { ref }}$ (red-dashed line) reaching it after 40 seconds.

The trajectories of $\xi_{2}$ (blue solid line); $\xi_{2, \text { ez }}$ (green solid line); and $\xi_{2 \text {,ref }}$ (red dashed line) are shown in Figure 2. Variable $\xi_{2}$ follows the sacrificed variable $\xi_{2, \mathrm{ez}}$, and after 60 seconds, both variables follow the reference $\xi_{2 \text {,ref }}$. In this way, the tracking errors $e_{1}$ and $e_{3}$ tend to zero. This remarks the performance of the proposed controller.

Figure 3 shows the trajectory of variable 3 . Variable $\xi_{3}$ (blue line) perfectly follows $\xi_{3 \text {,ref }}$ (red-dashed line) without undesirable oscillations.

Figures 4 and 5 present the control actions $u_{1}$ and $u_{2}$, respectively. Its initial values are $u_{1}(0)=u_{2}(0)=0$, 


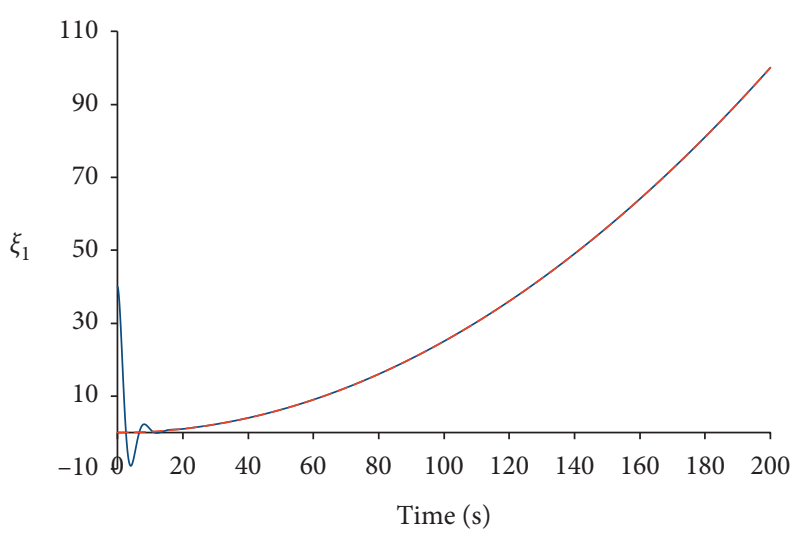

$$
\begin{aligned}
& -\xi_{1} \\
& ---\xi_{1, \text { ref }}
\end{aligned}
$$

Figure 1: Trajectory tracking of $\xi_{1}$.

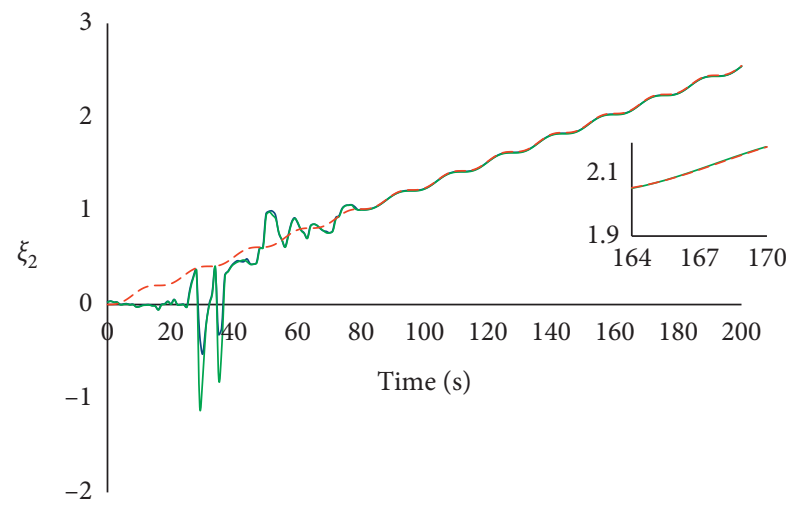

$$
\begin{aligned}
& -\xi_{2} \\
& -\xi_{2, \mathrm{ez}} \\
& --\xi_{2, \text { ref }}
\end{aligned}
$$

FIgURE 2: Trajectory tracking of $\xi_{2}$.

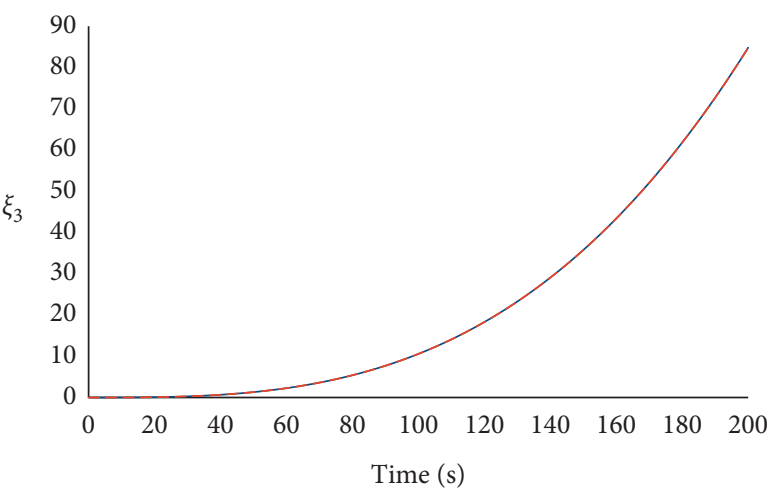

$$
\begin{aligned}
& -\xi_{3} \\
& ---\xi_{3, \text { ref }}
\end{aligned}
$$

FIgURE 3: Trajectory tracking of $\xi_{3}$.

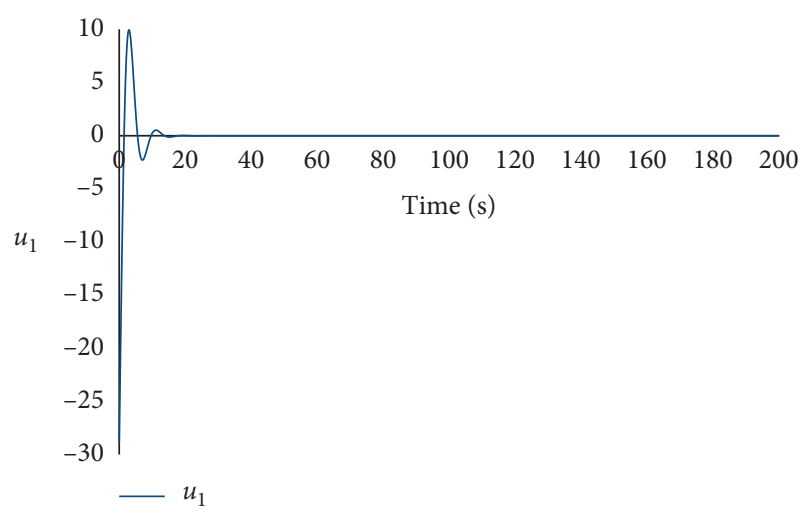

Figure 4: Control action $u_{1}$.

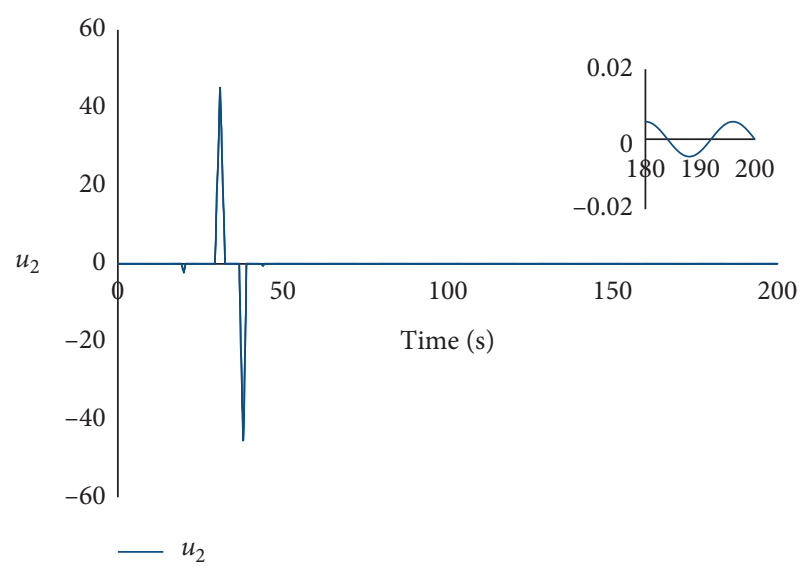

Figure 5: Control action $u_{2}$.

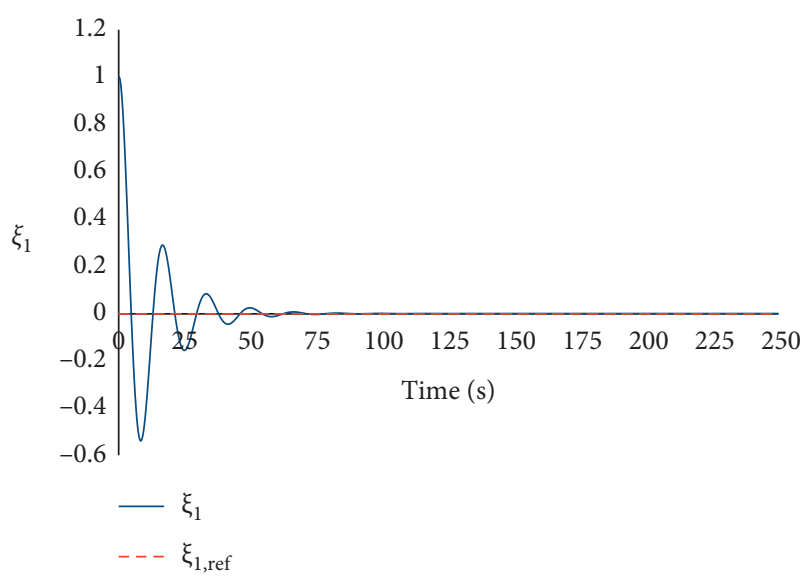

Figure 6: Trajectory of $\xi_{1}$.

respectively. The first one, $u_{1}$, evolves quickly from 0 to $10^{-4}$ remaining constant. The second one, $u_{2}$, follows a sine wave path with mean 0 , amplitude 0.8 , and frequency $1 / 8 \pi$.

3.2. Positioning Test. The closed-loop system (1)-(3) and (29) $-(31)$ is simulated considering $\xi_{1}(0)=1 ; \xi_{2}(0)=0$; and $\xi_{3}(0)=10^{-3}$ as the initial state. The controller parameters are $k_{10}=0.15 ; k_{11}=0.15 ; k_{20}=0.45 ; k_{21}=0.45 ; k_{30}=0.95$; 

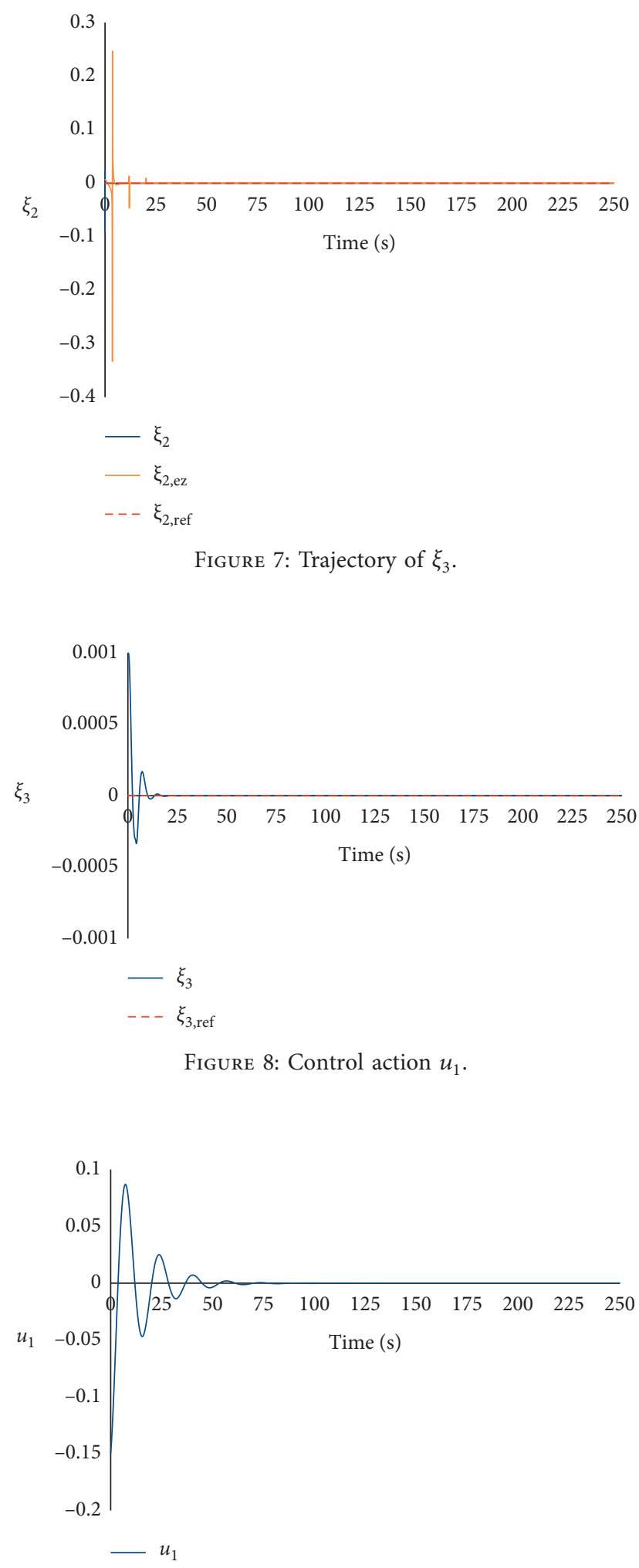

Figure 9: Trajectory of $\xi_{2}$.

and $k_{31}=0.95$. Figures 6 and 7 show the trajectories of system variables $\xi_{1}$ and $\xi_{3}$ (blue solid lines). The red and magenta dashed lines indicate the zero reference. Figure 8 presents the control action $u_{1}$, calculated by (30). In Figure 9, variables $\xi_{2}$ and $\xi_{2, \mathrm{ez}}$ are represented by a blue and orange

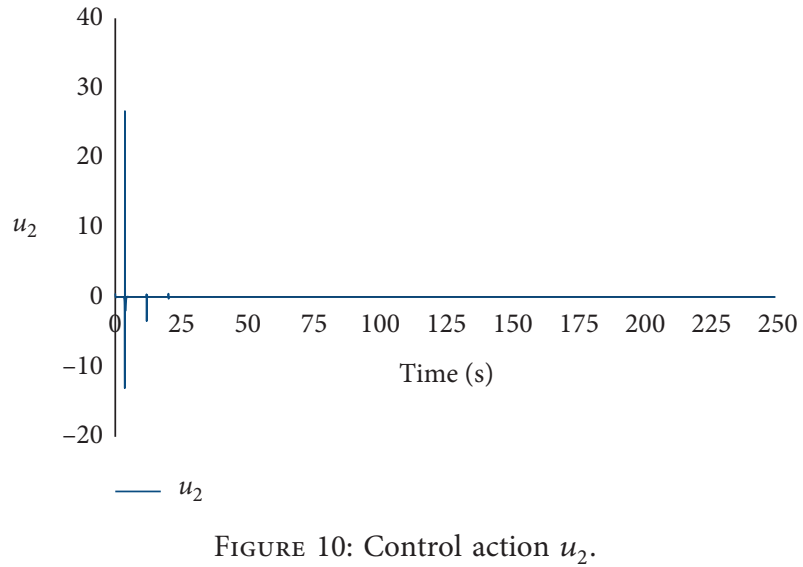

solid lines, respectively. Finally, Figure 10 displays the trajectory of the control action $u_{2}$ calculated by (31).

\section{Conclusion}

The major contribution of this paper is to extend the LABC technique for trajectory tracking and positioning of a second-order nonholonomic system. This controller is based on determining the conditions for which the system model has an exact solution. Knowing the model equations, it is necessary only to fix the desired trajectory to find the control action. Variables 1 and 3 were set as references, while variable 2 is the sacrificed one to ensure that the tracking errors go to zero. An important advantage of the proposed controller is the low computational load of the required mathematical operations. The stability of the system is demonstrated by applying the Khalil lemma [24].

Simulation results show the performance of the proposed control algorithm. The controller parameters were empirically tuned. For trajectory tracking, these parameters ensure that the system variables quickly reach the reference and follow it without undesirable oscillations. In the case of the positioning problem, these parameters were tuned in such a way variable $\xi_{3}$ goes to zero more quickly than $\xi_{1}$.

\section{Data Availability}

The data used to support the finding of this study are included within the article.

\section{Conflicts of Interest}

The authors declare that there are no conflicts of interest regarding the publication of this paper.

\section{Acknowledgments}

The authors thank the Institute of Chemical Engineering of the National University of San Juan, Argentina, Planta Piloto de Ingeniería Química (PLAPIQUI-National University of the South), Argentina, and Consejo Nacional de Investigaciones Científicas y Técnicas (CONICET, National Council for Scientific Research), Argentina. This work was partially funded by Consejo Nacional de Investigaciones 
Científicas y Técnicas (CONICET), Universidad Nacional de San Juan (UNSJ), and Universidad Nacional del Sur (UNS), Argentina.

\section{References}

[1] G. He, C. Zhang, W. Sun, and Z. Geng, "Stabilizing the second-order nonholonomic systems with chained form by finite-time stabilizing controllers," Robotica, vol. 34, no. 10, pp. 2344-2367, 2016.

[2] N. P. I. Aneke, Control of underactuated mechanical systems, Ph.D. dissertation, Department of Mechanical Engineering, Eindhoven University of Technology, Eindhoven, The Netherlands, 2003.

[3] R. W. Brockett, R. S. Millman, and H. J. Sussmann, "Asymptotic stability and feedback stabilization," in Differential Geometric Control Theory, pp. 2961-2963, Birkhäuser, Basel, Switzerland, 1983.

[4] N. P. I. Aneke, H. Nijmeijer, and A. G. de Jager, "Trajectory tracking by cascaded backstepping control for a second-order non-holonomic mechanical system,", " in Nonlinear Control in the Year 2000. Lecture Notes in Control and Information Sciences, A. Isidori, F. Lamnabhi-Lagarrigue, and W. Respondek, Eds., vol. 258, pp. 35-49, Springer, Paris, France, 2000.

[5] N. P. I. Aneke, H. Nijmeijer, and A. G. de Jager, "Tracking control of second-order chained form systems by cascaded backstepping," International Journal of Robust and Nonlinear Control, vol. 13, no. 2, pp. 95-115, 2003.

[6] Z. P. Yuan, Z. P. Wang, and Q. J. Chen, "Trajectory tracking control of a nonholonomic mobile robot," in Proceedings of the IEEE ICCA 2010, pp. 2207-2211, Xiamen, China, June 2010.

[7] N. Adhikary and C. Mahanta, "Integral backstepping sliding mode control for underactuated systems: swing-up and stabilization of the cart-pendulum system," ISA Transactions, vol. 52, no. 6, pp. 870-880, 2013.

[8] Y.-P. Tian and K.-C. Cao, “Time-varying linear controllers for exponential tracking of non-holonomic systems in chained form," International Journal of Robust and Nonlinear Control, vol. 17, no. 7, pp. 631-647, 2007.

[9] A. Astolf, "Discontinuous control of nonholonomic systems," Systems \& Control Letters, vol. 27, no. 1, pp. 37-45, 1996.

[10] Y. Nakamura and R. Mukherjee, "Nonholonomic path planning of space robots via a bidirectional approach," IEEE Transactions on Robotics and Automation, vol. 7, no. 4, pp. 500-514, 1991.

[11] M. Asif, M. J. Khan, and N. Cai, "Adaptive sliding mode dynamic controller with integrator in the loop for nonholonomic wheeled mobile robot trajectory tracking," International Journal of Control, vol. 87, no. 5, pp. 964-975, 2014.

[12] L. Yang and J. Y. Yang, "Stabilization for a class of nonholonomic perturbed systems via robust adaptive sliding mode control," in Proceedings of the American Control Conference, pp. 1178-1183, Baltimore, MD, USA, June 2010.

[13] N. Chen, F. Song, G. Li, X. Sun, and C. Ai, "An adaptive sliding mode backstepping control for the mobile manipulator with nonholonomic constraints," Communications in Nonlinear Science and Numerical Simulation, vol. 18, no. 10, pp. 2885-2899, 2013.

[14] M. A. Fairus, Z. Mohamed, M. N. Ahmad, and W. S. Loi, "LMI-based multi objective integral sliding mode control for rotary inverted pendulum system under load variations," Journal Teknologi, vol. 73, pp. 125-137, 2015.

[15] M. Sarfraz and F. ur Rehman, "Adaptive sliding mode control for a second order nonholonomic planar four-link UMS," in Proceedings of the 2017 13th International Conference on Emerging Technologies (ICET), pp. 1-6, Islamabad, Pakistan, July 2017.

[16] S. Gustavo, S. M. Emanuel, and A. Pedro, Linear Algebra Based Controller - Design and Applications, Springer International Publishing, Cham, Switzerland, 2020.

[17] G. Scaglia, E. Serrano, A. Rosales, and P. Albertos, "Tracking control design in nonlinear multivariable systems: robotic applications," Mathematical Problems in Engineering, vol. 2019, Article ID 8643515, 15 pages, 2019.

[18] G. Scaglia, V. Mut, A. Rosales, and O. Quintero, "Tracking control of a mobile robot using linear interpolation," IMAACA07, vol. 1, pp. 11-15, 2007.

[19] A. Rosales, G. Scaglia, V. Mut, and F. Di Sciascio, "Trajectory tracking of mobile robots in dynamic environments-a linear algebra approach," Robotica, vol. 27, no. 7, pp. 981-997, 2009.

[20] M. E. Serrano, S. A. Godoy, L. Quintero, and G. J. Scaglia, "Interpolation based controller for trajectory tracking in mobile robots," Journal of Intelligent \& Robotic Systems, vol. 86, no. 3-4, pp. 569-581, 2017.

[21] M. E. Serrano, G. J. E. Scaglia, S. A. Godoy, V. Mut, and O. A. Ortiz, "Trajectory tracking of underactuated surface vessels: a linear algebra approach," IEEE Transactions on Control Systems Technology, vol. 22, no. 3, pp. 1103-1111, 2014.

[22] M. E. Serrano, G. J. E. Scaglia, F. A. Cheein, V. Mut, and O. A. Ortiz, "Trajectory-tracking controller design with constraints in the control signals: a case study in mobile robots," Robotica, vol. 33, no. 10, pp. 2186-2203, 2015.

[23] G. Scaglia, P. M. Aballay, M. E. Serrano, O. A. Ortiz, M. Jordan, and M. D. Vallejo, "Linear algebra based controller design applied to a bench-scale oenological alcoholic fermentation," Control Engineering Practice, vol. 25, pp. 66-74, 2014.

[24] H. K. Khalil, Nonlinear Systems, Prentice-Hall, Upper Saddle River, NJ, USA, 2nd edition, 1996. 